DEUTSCHES LITERATUR-LEXIKON ACHTUNDDREISSIGSTER BAND 



\section{DEUTSCHES}

\section{LITERATUR-LEXIKON}

BIOGRAPHISCH-BIBLIOGRAPHISCHES HANDBUCH

BEGRÜNDET VON WILHELM KOSCH

DRITTE, VÖLLIG NEU BEARBEITETE AUFLAGE

ACHTUNDDREISSIGSTER BAND:

ZIMMER - ZYX

WISSENSCHAFTLICHER BEIRAT:

WOLFGANG ACHNITZ, LUTZ HAGESTEDT, MARIO MÜLLER, CLAUS-MICHAEL ORT, REIMUND B. SDZUJ

DE GRUYTER 
Florian Altenhöfer, M. A., München; Dr. Dörthe Buchhester, Hildesheim;

Mag. Sabina Foidl, München; Prof. Dr. Lutz Hagestedt, Rostock; Bruno Jahn, München;

Dr. Jacob Klingner, Berlin; Manfred Knedlik, M. A., Neumarkt; Dr. Mike Malm, München; Dr. Christoph Michel, Freiburg i. Br.; PD Dr. Mario Müller, Hildesheim; Prof. Dr. Hans Pörnbacher, Wildsteig; PD Dr. Reimund B. Sdzuj, Greifswald; Dr. Christine Stridde, Zürich; Dr. Maria Tischler, München; Paul Tischler, Dipl.-Germ., München; Dr. Volker Zapf, München

\title{
WISSENSCHAFTLICHER BEIRAT
}

Mittelalter: PD Dr. Wolfgang Achnitz, Universität Münster Frühe Neuzeit: PD Dr. Reimund B. Sdzuj, Universität Frankfurt/M., Universität Greifswald I8. Jahrhundert: PD Dr. Mario Müller, Universität Hildesheim I9. Jahrhundert: Prof. Dr. Claus-Michael Ort, Universität Kiel 20. und 2I. Jahrhundert: Prof. Dr. Lutz Hagestedt, Universität Rostock

REDAKTIONELLE LEITUNG

Bruno Jahn

REDAKTIONSSCHLUSS

30. September 2018

\author{
ISBN 978-3-II-056887-5 \\ e-ISBN (PDF) 978-3-II-057005-2 \\ e-ISBN (EPUB) 978-3-II-058895-O
}

\section{Bibliographische Information Der Deutschen Nationalbibliothek}

Die Deutsche Nationalbibliothek verzeichnet diese Publikation in der Deutschen Nationalbibliographie; detaillierte bibliographische Daten sind im Internet über http://dnb.d-nb.de abrufbar.

(C) Copyright 2019 Walter de Gruyter GmbH, Berlin/Boston

Satz: bsix information exchange $\mathrm{GmbH}$, Braunschweig

Druck: Strauss GmbH, Mörlenbach

@ Gedruckt auf säurefreiem Papier

Printed in Germany

www.degruyter.com 